\title{
Credibility Test for Frequency Estimation of Sinusoid Using Chebyshev's Inequality
}

\author{
Hu Guobing, ${ }^{1,2}$ Xu LiZhong, ${ }^{2}$ Gao Yan, ${ }^{1}$ and Wu Shanshan ${ }^{1}$ \\ ${ }^{1}$ Nanjing College of Information Technology, Nanjing 210023, China \\ ${ }^{2}$ Hohai University, Nanjing 210098, China \\ Correspondence should be addressed to Hu Guobing; guobinghu@163.com
}

Received 16 August 2014; Revised 19 November 2014; Accepted 19 November 2014; Published 4 December 2014

Academic Editor: Kui Fu Chen

Copyright (c) $2014 \mathrm{Hu}$ Guobing et al. This is an open access article distributed under the Creative Commons Attribution License, which permits unrestricted use, distribution, and reproduction in any medium, provided the original work is properly cited.

\begin{abstract}
Estimation of sinusoid frequency is a key research problem related to radar, sonar, and communication systems. The results of numerous investigations on frequency estimation have been reported in the literature. Nevertheless, to the best of our knowledge, none of them have dealt with credibility evaluation, which is used to decide whether an individual frequency estimate of the sinusoid is accurate or not. In this study, the credibility problem is modeled as a hypothesis test based on Chebyshev's inequality (CI). The correlation calculated from the received signal and the reference signal generated according to the frequency estimate is used as a test statistic. A threshold is determined based on CI, and the analytical expression for the frequency estimation credibility detection performance is derived. Simulations show that the proposed method performs well even at low signal-to-noise ratios.
\end{abstract}

\section{Introduction}

Frequency estimation of a sinusoid signal is an important problem concerning applications related to commercial and military signal processing systems. For some methods, estimation of frequency is the precondition for estimating the other parameters of sinusoid signals [1] as well as frequency estimation of modulated signals [2,3]. Many algorithms have been proposed for estimation of frequency from received signals [4-12]. Performance evaluation of frequency estimation algorithms is also a key operation in practical signal processing systems and can be considered from two points of view. Algorithm designers focus on the overall statistical performance that can be evaluated by comparing the mean square error with the Cramér-Rao lower bound (CRLB). On the other hand, users consider the credibility (or confidence) of individual frequency estimation important. In a noncooperative context, especially at low signal-to-noise (SNR) ratios, it is important to decide whether an individual frequency estimate is accurate or not when the true value of the frequency is unknown at the receiver side. For example, in the pulse sorting system, pulse radio frequency is one of the five key parameters that are pulse duration $(\mathrm{PD})$, pulse amplitude (PA), pulse radio frequency (RF), angle of arrival
(AOA), and time of arrival (TOA). Therefore, the sorting performance may be affected by the accuracy and credibility of pulse radio frequency. As another example in electronic intelligence (Elint), it is helpful for the users to determine the parameter limit and to remove the outlier estimates by using the credibility checking of each estimate [13].

Recently, investigations conducted have been devoted to the confidence evaluation of blind modulation recognition results to enhance the reliability of the overall signal processing units and conserve both software and hardware resources. The confidence measurement of modulation recognition becomes the key output information of the signal processing system in military applications and is used to identify unknown radar signals [14]. As IEEE 1990.6 for cognitive radio (CR) [15] expressed, the modulation recognition confidence rating is regarded as additional output information in some civilian signal processing devices; an example is Agilent's option MR1 for E3238S signal detection and monitoring systems. Nevertheless, the detailed method of evaluating the reliability of modulation recognition is not described in both [10,11]. Fehske et al. [16] defined the half value of the maximum and the second maximum output of the back propagation- (BP-) based classifier as the confidence metric of modulation classifying results for 
CR. Lin and Liu [17] proposed a confidence measurement method based on the information entropy to measure the confidence of modulation recognition results in single-input and single-output (SISO) as well as multiple-input multipleoutput (MIMO) channels of CR. Still, credibility evaluation of frequency estimation of a sinusoid remains an inadequately addressed research problem.

In this paper, we propose a method to automatically decide the credibility of an individual frequency estimate of a sinusoid without any a priori knowledge about the parameters of the received signal. Section 2 of this paper presents the sinusoid signal model and a hypothesis test for credibility evaluation. The null hypothesis is defined as that when the absolute frequency estimation error of the sinusoid is less than a quarter of a discrete frequency interval and the alternative one is the contrary. In Section 3, the statistic is defined by calculating the magnitude of the correlation between the received signal and the reference signal generated according to a frequency estimator. If the absolute estimation error is less than a quarter of the discrete frequency interval, it shows that the mean of the correlation under the null hypothesis is different from that under the alternative hypothesis. By using this property, Section 4 presents a decision rule for the credibility test for frequency estimation of sinusoid based on Chebyshev's inequality (CI). Finally, Section 5 summarizes the proposed algorithm, and Section 6 reports the simulation results.

\section{Signal Model and Basic Assumptions}

2.1. Signal Model. A complex sinusoid contaminated by noises can be described by the following signal model:

$$
\begin{aligned}
x(n) & =s(n)+w(n) \\
& =A \exp \left[j\left(2 \pi f_{0} n \Delta t+\theta\right)\right]+w(n), \\
& 0 \leq n \leq N-1,
\end{aligned}
$$

where $A, f_{0}$, and $\theta$ denote, respectively, the amplitude, carrier frequency, and initial phase of the sinusoid signal $s(n) . \Delta t$ is the discrete sampling interval, and $N$ corresponds to the length of the samples. The additive noise $w(n)$ is supposed to be a white complex Gaussian process with a zero mean and variance $\sigma^{2}$ whose real and imaginary parts are independent of each other.

\subsection{Hypothesis Model for Credibility Evaluation. In the non-} cooperative environment, both the modulation format and signal parameters are unknown at the receiver side. Generally, processing of the sinusoid is performed in two main steps: modulation recognition and carrier frequency estimation. For a certain processing cycle, credibility evaluation of the frequency estimator is aimed at detecting whether the individual frequency estimate is accurate or not. In practice, for the widely used fast Fourier transform- (FFT-) based estimators, if the signal-to-noise ratio (SNR) is greater than the moderate threshold, the maximum absolute bias of the frequency estimation $(|\Delta f|)$ is less than a quarter of the discrete sampling frequency interval $(\Delta F)[18]$. Therefore, we describe the credibility assessment as the following hypothesis test:

$$
\begin{aligned}
& H_{0}:|\Delta f| \leq 0.25 \Delta F \\
& H_{1}:|\Delta f|>0.25 \Delta F
\end{aligned}
$$

\section{Statistic Selection and Analysis}

3.1. Feature Analysis. Assuming that the observed signal is a single-tone sinusoid, the reference signal can be constructed by the sinusoid model as follows:

$$
y(n)=\exp \left(-j 2 \pi \widehat{f}_{0} n \Delta t\right), \quad 0 \leq n \leq N-1,
$$

where $\widehat{f}_{0}$ is estimated by the maximum likelihood (ML) method or the other suboptimal estimators. The correlation between the observed signal $x(n)$ and the reference signal $y(n)$ can be expressed as

$$
Z=\sum_{m=0}^{N-1}[s(m)+w(m)] y(m)=s+w
$$

where $s$ and $w$ are the signal part and noise part of $Z$, respectively. The signal part $s$ can be further derived as

$$
s=A \frac{\sin (\pi \Delta f \Delta t N)}{\sin (\pi \Delta f \Delta t)} e^{j \beta},
$$

where $\beta=\pi(N-1) \Delta f \Delta t+\theta$ is the phase of $s$. Letting $\delta=$ $\Delta f / \Delta F$, the magnitude of $s$ is given by

$$
s=A\left|\frac{\sin (\pi \Delta f \Delta t N)}{\sin (\pi \Delta f \Delta t)}\right| \approx N A \operatorname{sinc}(\delta),
$$

where $\delta$ is the factor of the frequency estimation error and $\operatorname{sinc}(x)=\sin \pi x / \pi x$ is the sinc function.

Rewrite $Z$ to the algebraic form as

$$
Z=Z_{R}+j Z_{I}
$$

where $Z_{R}, Z_{I}$ are the real and imaginary parts of $Z$, respectively. They are independent Gaussian distributions. Now, the mean and variance of $Z_{R}$ and $Z_{I}$ can be expressed as follows:

$$
\begin{gathered}
E\left(Z_{R}\right)=\mu_{z_{R}}=N A|\operatorname{sinc}(\delta)| \cos \beta, \\
E\left(Z_{I}\right)=\mu_{z_{I}}=N A|\operatorname{sinc}(\delta)| \sin \beta, \\
D\left(Z_{R}\right)=D\left(Z_{I}\right)=\frac{1}{2} D(Z)=\frac{N \sigma^{2}}{2}=\sigma_{z}^{2} .
\end{gathered}
$$

For the appropriate frequency estimator, if the SNRs are above a certain threshold $[5,14]$, the absolute estimation error is less than the discrete sampling frequency interval $\Delta F$; that is, $0 \leq \delta \leq 1$. Consider the magnitude of the mean of $Z$ as

$$
|E(Z)|=|s|=\sqrt{\mu_{z_{R}}^{2}+\mu_{z_{I}}^{2}}=N A \operatorname{sinc}(\delta) .
$$




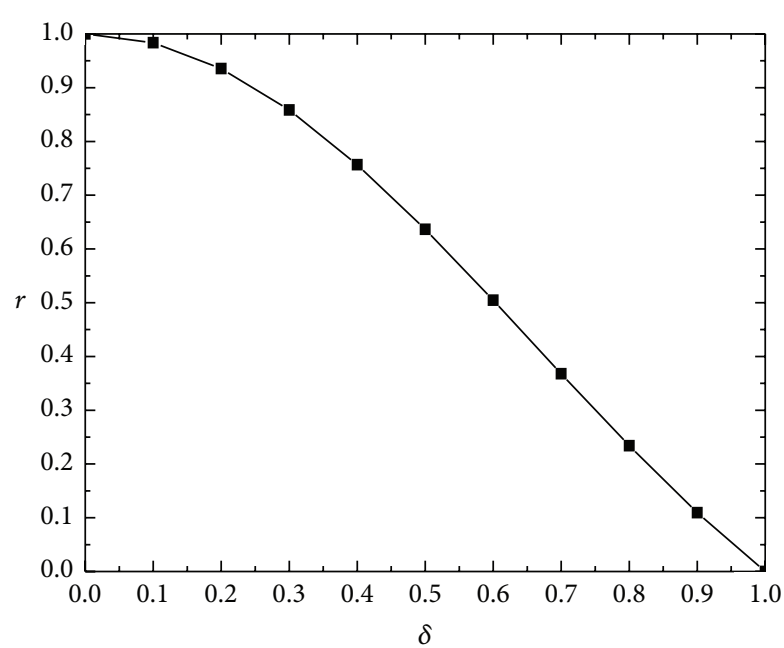

FIGURE 1: Relationship between $r$ and $\delta$.

Under hypothesis $H_{0}$, assuming that the absolute error is relatively small, that is, $|\Delta f| \leq 0.25 \Delta F$ and $\delta \leq 0.25$, it follows that

$$
\left|E\left(Z_{0}\right)\right|=N A|\operatorname{sinc}(\delta)| \geq N A \operatorname{sinc}(0.25) \approx 0.9003 N A \text {. }
$$

Under hypothesis $H_{1}$, because of the low SNR or other possible reasons, the maximum magnitude of the observed signals' spectrums might not be located in the correct point or the discrete frequency interval and the estimation error $\Delta f$ is larger than $\Delta F / 4$; that is, $\delta \geq 0.25$. Thus,

$$
\left|E\left(Z_{1}\right)\right|=N A \operatorname{sinc}(\delta) \leq 0.9003 N A \text {. }
$$

In order to explain the relationship between the frequency estimation error and the magnitude of the mean of $Z$, we define the ratio as

$$
r=\frac{|E(Z)|_{\delta}}{|E(Z)|_{0}} \approx \operatorname{sinc}(\delta)
$$

where $|E(Z)|_{\delta}$ is the magnitude of the mean of $Z$ at a given $\delta$. According to the sinc function, the ratio decreases monotonically with the frequency estimating error factor $\delta$ supposing $0 \leq \delta \leq 1$. As $\Delta f$ increases, $\delta$ increases and the ratio $r$ decreases. This relationship is shown in Figure 1. For $\delta \leq 0.25$, the ratio $r>0.9003$ and it decreases slowly departing from 1 with the increase of $\delta$. For $\delta>0.25$, the ratio decreases dramatically. Hence, we can infer the absolute error of certain frequency estimation depending on the ratio $r$ and obtain the credibility metric of the estimation.

In order to facilitate convenient handling of the formula derivation, the statistic used to decide $H_{0}$ and $H_{1}$ can be defined as follows:

$$
V=\frac{|Z|^{2}}{\sigma_{z}}
$$

Clearly, the random variable $V$ under $H_{i}, i=0,1$, follows a standard noncentral law and its contribution can be represented as [19]

$$
p_{V \mid H_{i}}(v)= \begin{cases}\frac{1}{2} \exp \left[-\frac{1}{2}\left(v^{2}+\lambda_{i}^{2}\right)\right] I_{0}\left(\sqrt{\lambda_{i} v}\right), & v \geq 0 \\ 0, & v<0\end{cases}
$$

where $I_{0}(x)$ is the zero-order modified Bessel function and $\lambda_{i}=\left(\mu_{z_{R i}}^{2}+\mu_{z_{I i}}^{2}\right) / \sigma_{z}^{2}$ denotes the noncentrality.

Under the assumption relating to $H_{0}$, that is, $\delta \leq 0.25$, we obtain

$$
\lambda_{0} \geq \lambda_{t}=\frac{2[N A \operatorname{sinc}(0.25)]^{2}}{N \sigma^{2}} \approx 1.6211 N \mathrm{SNR}
$$

where $\lambda_{t}$ is the noncentrality parameter when $\delta=0.25$. Similarly, under the $H_{1}$ assumption, we obtain

$$
\lambda_{1}=2[\operatorname{sinc}(\delta)]^{2} N \mathrm{SNR}<\lambda_{t} .
$$

From the above discussion, we find that, under each assumption, $H_{0}$ or $H_{1}$, the statistic $V$ follows a standard noncentral law with different parameters. Therefore, the hypothesis test defined by (2) can be transformed to a parameter test involving $\lambda$ that is given by

$$
H_{0}: \lambda \geq \lambda_{t}, \quad H_{1}: \lambda<\lambda_{t} .
$$

3.2. Decision Rule and the Threshold. We first consider the degradation form of (17) given as

$$
H_{0}^{\prime}: \lambda=\lambda_{t}, \quad H_{1}^{\prime}: \lambda \neq \lambda_{t} .
$$

Consequently, letting $c=2+\lambda, b=\lambda / c$, the statistic $V$ can be approximated to a Gaussian distribution by $W=(V / c)^{1 / 3}$ [20] whose mean and variance are given by

$$
E(W)=1-\frac{2(1+b)}{9 c}, \quad D(W)=\frac{2(1+b)}{9 c},
$$

respectively. Thus, the standard variable

$$
U=\frac{W-E(W)}{\sqrt{D(W)}} \sim N(0,1)
$$

and the hypothesis test expressed by (17) can be simplified by

$$
H_{0}^{\prime}: \mu_{U}=0, \quad H_{1}^{\prime}: \mu_{U} \neq 0,
$$

where $\mu_{U}$ denotes the mean of $U$.

As the means of $U$ between the two assumptions are different, CI can be used as the decision rule to perform the hypothesis test. Hence, we write CI as

$$
P\{|U| \leq \varepsilon\} \geq 1-\frac{1}{\varepsilon^{2}},
$$

where $\varepsilon>0$ is a certain real number. As $U$ approximately follows a standard Gaussian law, the probability of the samples located in the range of threefold the standard error is 




FIgURe 2: Distribution of the statistic $U$ for $\delta=0.1,0.25,0.4$ at $\mathrm{SNR}=0 \mathrm{~dB}$. The carrier frequency is set to $19.081 \mathrm{MHz}$, the length of received samples is 1024 , the initial phase is $\pi / 6$, and the number of simulations is 1000 .

greater than 0.9973 and we can adjust $\varepsilon$ to obtain the desired probability. Hence, we decide $H_{1}^{\prime}$ if

$$
-\varepsilon \leq U \leq \varepsilon .
$$

Consequently, the hypothesis test of (2) can be rewritten as

$$
H_{0}: \mu_{U} \geq 0, \quad H_{1}: \mu_{U}<0 .
$$

Therefore, we decide $H_{1}$ if

$$
U \geq \gamma=-\varepsilon,
$$

where $\gamma$ is the threshold. The probability of false alarm can be defined as

$$
P_{r}\left\{U<\gamma ; H_{0}\right\} \approx \Phi(\gamma)=\alpha,
$$

where $\Phi(x)=(1 / \sqrt{2 \pi}) \int_{-\infty}^{x} e^{-t^{2} / 2} d t$ is the distribution function of a standard Gaussian variable and $\alpha$ is a given false alarm. Taking the inverse function of (26) leads to the threshold

$$
\gamma=\Phi^{-1}(\alpha)
$$

where $\Phi^{-1}(x)$ denotes the inverse of the distribution function of a standard Gaussian probability density function (pdf).

Figure 2 shows the histogram and the fitted pdfs of the statistic $U$ for different frequency estimator factors. It can be seen that, under a certain SNR, the proposed statistic based on $\mathrm{CI}$ approximated to the standard Gaussian and the selected threshold can be used to distinguish $H_{0}$ and $H_{1}$ assumptions effectively.

\section{Algorithm Summary}

The proposed credibility test algorithm is composed of the following steps.

(1) Estimation of the frequency of the signal by using a certain estimation method.

(2) Construction of the reference signal $y(n)$ with (3) by using the estimated frequency $\widehat{f}_{0}$.

(3) Computation of the correlation between the received signal and the reference signal and contraction of the statistic $U$ with (20).

(4) Computation of the test threshold $\gamma$ from the probability of false alarm with (27).

(5) Evaluation of the credibility of frequency estimation by comparing the statistic $U$ to the threshold $\gamma$ with (25). The hypothesis $H_{0}$ is chosen if $U<\gamma$. Otherwise $H_{1}$ is accepted.

\section{Performance Analysis}

Under $H_{0}^{\prime}$ assumption with $\lambda=\lambda_{t}$, the statistic defined in (20) is expressed as

$$
U_{H_{0}^{\prime}}^{\lambda_{t}}=\frac{\left(V / c_{t}\right)^{1 / 3}-\left[1-(2 / 9)\left(1+b_{t}\right) / c_{t}\right]}{\sqrt{(2 / 9)\left(1+b_{t}\right) / c_{t}}} \sim N(0,1),
$$

where $c_{t}=2+\lambda_{t}$ and $b=\lambda_{t} / c_{t}$, respectively.

For $\lambda \geq \lambda_{t}$, the statistic is

$$
U_{H_{0}}^{\lambda}=\frac{(V / c)^{1 / 3}-[1-(2 / 9)(1+b) / c]}{\sqrt{(2 / 9)(1+b) / c}} \sim N(0,1) .
$$

We note that, for $\lambda>\lambda_{t}$, it can be rewritten as

$$
\begin{aligned}
U_{H_{0}}^{\lambda_{t}}= & \frac{\left(V / c_{t}\right)^{1 / 3}-\left[1-(2 / 9)\left(1+b_{t}\right) / c_{t}\right]}{\sqrt{(2 / 9)\left(1+b_{t}\right) / c_{t}}} \\
= & \frac{(V / c)^{1 / 3}-\varsigma^{1 / 3}\left[1-(2 / 9)\left(1+b_{t}\right) / c_{t}\right]}{\varsigma^{1 / 3} \sqrt{(2 / 9)\left(1+b_{t}\right) / c_{t}}} \\
= & \left(\left(\frac{V}{c}\right)^{1 / 3}-\left[1-\frac{(2 / 9)(1+b)}{c}\right]\right. \\
& +\left[1-\frac{(2 / 9)(1+b)}{c}\right] \\
& \left.-\varsigma^{1 / 3}\left[1-\frac{(2 / 9)\left(1+b_{t}\right)}{c_{t}}\right]\right) \\
& \times\left(\varsigma^{1 / 3} \sqrt{\frac{(2 / 9)\left(1+b_{t}\right)}{c_{t}}}\right)^{-1},
\end{aligned}
$$

where $\varsigma=c_{t} / c$ and $\varsigma \geq 1$. According to the property of the Gaussian distribution, the statistic is given by

$$
U_{H_{0}}^{\lambda_{t}} \sim N\left(\mu_{H_{0}}^{\lambda_{t}}, \sigma_{H_{0}}^{\lambda_{t}}\right) \text {, }
$$


where

$$
\begin{aligned}
\mu_{H_{0}}^{\lambda_{t}} & =E\left(U_{H_{0}}^{\lambda_{t}}\right) \\
& =\frac{1-(2 / 9)(1+b) / c-\varsigma^{1 / 3}\left[1-(2 / 9)\left(1+b_{t}\right) / c_{t}\right]}{\varsigma^{1 / 3} \sqrt{(2 / 9)\left(1+b_{t}\right) / c_{t}}}
\end{aligned}
$$

and the variance is

$$
\sigma_{H_{0}}^{\lambda_{t}}=\sqrt{D\left(U_{H_{0}}^{\lambda_{t}}\right)}=\varsigma^{-1 / 3} \sqrt{\frac{(1+b) / c}{\left(1+b_{t}\right) / c_{t}}} .
$$

Hence, we obtain the probability of false alarm as

$$
\begin{aligned}
P_{\mathrm{FA}} & =P\left\{U_{0}^{\lambda_{t}} \leq \gamma \mid H_{0}\right\} \\
& =\int_{-\infty}^{\gamma} \frac{1}{\sqrt{2 \pi} \sigma_{H_{0}}^{\lambda_{t}}} \exp \left[-\frac{\left(x-\mu_{H_{0}}^{\lambda_{t}}\right)^{2}}{2\left(\sigma_{H_{0}}^{\lambda_{t}}\right)^{2}}\right] d x \\
& \approx \Phi\left(\frac{\gamma-\mu_{H_{0}}^{\lambda_{t}}}{\sigma_{H_{0}}^{\lambda_{t}}}\right) .
\end{aligned}
$$

Similarly, under $H_{1}$ assumption with $\lambda<\lambda_{t}$, the statistic

$$
\begin{aligned}
U_{H_{1}}^{\lambda_{t}}= & \frac{\left(V / c_{t}\right)^{1 / 3}-\left[1-(2 / 9)\left(1+b_{t}\right) / c_{t}\right]}{\sqrt{(2 / 9)\left(1+b_{t}\right) / c_{t}}} \\
= & \left(\left(\frac{V}{c}\right)^{1 / 3}-\left[1-\frac{(2 / 9)(1+b)}{c}\right]\right. \\
& +\left[1-\frac{(2 / 9)(1+b)}{c}\right] \\
& \left.-\xi^{1 / 3}\left[1-\frac{(2 / 9)\left(1+b_{t}\right)}{c_{t}}\right]\right) \\
& \times\left(\xi^{1 / 3} \sqrt{\left.\frac{(2 / 9)\left(1+b_{t}\right)}{c_{t}}\right)^{-1}},\right.
\end{aligned}
$$

where $\xi=c_{t} / c$ and $0 \leq \xi \leq 1$. From (35), $U_{H_{1}}^{\lambda_{t}} \sim N\left(\mu_{H_{1}}^{\lambda_{t}}, \sigma_{H_{1}}^{\lambda_{t}}\right)$ with the mean and variance are, respectively, given by

$$
\begin{aligned}
\mu_{H_{1}}^{\lambda_{t}}= & E\left(U_{H_{1}}^{\lambda_{t}}\right) \\
= & \left(1-\frac{(2 / 9)(1+b)}{c}\right. \\
& \left.-\xi^{1 / 3}\left[1-\frac{(2 / 9)\left(1+b_{t}\right)}{c_{t}}\right]\right) \\
& \times\left(\xi^{1 / 3} \sqrt{\left.\frac{(2 / 9)\left(1+b_{t}\right)}{c_{t}}\right)^{-1}},\right. \\
\sigma_{H_{1}}^{\lambda_{t}}= & \sqrt{D\left(U_{H_{1}}^{\lambda_{t}}\right)}=\xi^{-1 / 3} \sqrt{\frac{(1+b) / c}{\left(1+b_{t}\right) / c_{t}}}
\end{aligned}
$$

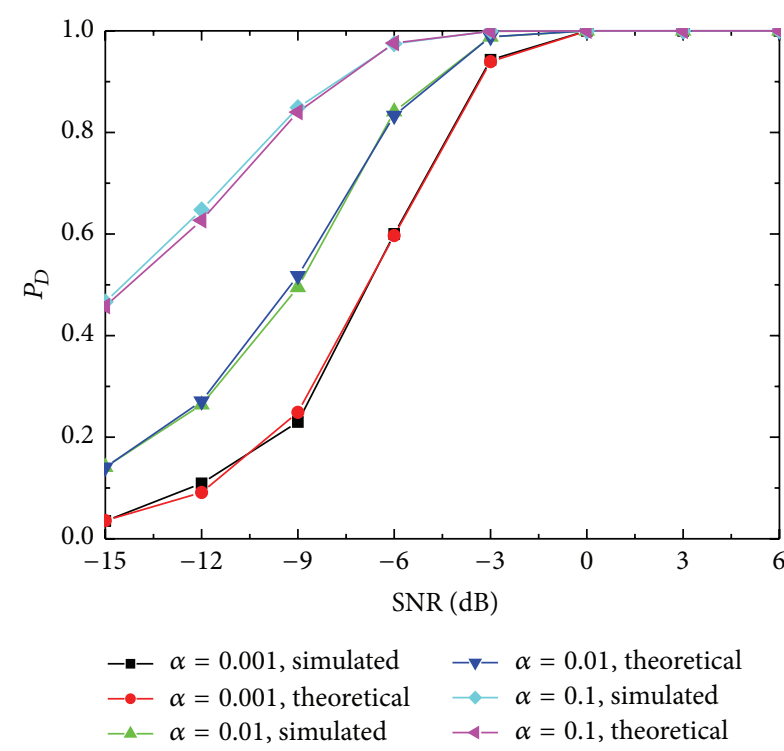

FIGURE 3: Effect of the false alarm probability on the detection probability obtained by the credibility test of a frequency estimate.

Thus, the detection probability can be expressed by

$$
\begin{aligned}
P_{D} & =\operatorname{Pr}\left\{U_{H_{1}}^{\lambda_{t}} \leq \gamma \mid H_{1}\right\} \\
& =\int_{-\infty}^{\gamma} \frac{1}{\sqrt{2 \pi} \sigma_{H_{1}}^{\lambda_{t}}} \exp \left[-\frac{\left(x-\mu_{H_{1}}^{\lambda_{t}}\right)^{2}}{2\left(\sigma_{H_{1}}^{\lambda_{t}}\right)^{2}}\right] d x \\
& \approx \Phi\left(\frac{\gamma-\mu_{H_{1}}^{\lambda_{t}}}{\sigma_{H_{1}}^{\lambda_{t}}}\right) .
\end{aligned}
$$

From (34) and (37), we observe that the detection performance depends largely on the parameter $\lambda$, which is the function of the number of samples received, the SNR, and the frequency estimation error factor.

\section{Simulation Results}

Monte Carlo simulations were carried out to study the behavior of the proposed algorithm in different environments. The simulations were aimed at detecting whether the absolute error of a certain estimated frequency is greater than a quarter of the discrete sampling frequency. Ten thousand Monte Carlo trials were performed for each condition. Monte Carlo trials were run in each of the following conditions: (1) the sinusoid signal contaminated by additive white Gaussian noises is received; (2) $\mathrm{SNR}=10 \log _{10} A^{2} / \sigma^{2}$; (3) the sampling frequency is set to $100 \mathrm{MHz}$ except in 6.6.

6.1. Influence of False Alarm. Figure 3 shows the detection behavior for the credibility test by using the CI method with respect to the false alarm and the comparisons with the values theoretically calculated using (37). The carrier frequency of the sinusoid is $19.801 \mathrm{MHz}$, while the sample size is 1024 . The 


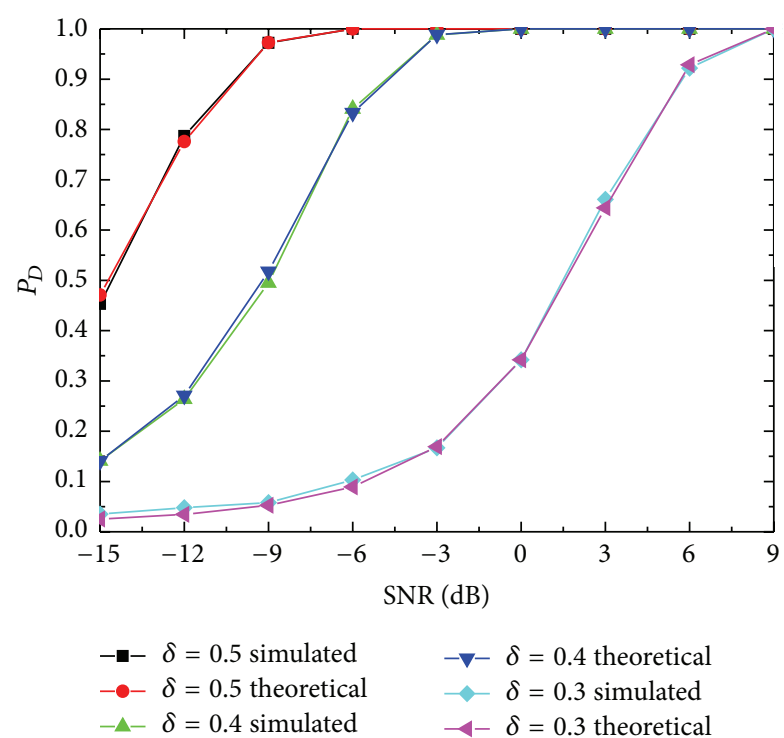

FIgURE 4: Effect of the frequency estimation error factor on the detection probability obtained by the credibility test of a frequency estimate.

initial phase is $\pi / 6$, and the frequency estimation error factor is 0.5 . The SNR is varied from $-15 \mathrm{~dB}$ to $6 \mathrm{~dB}$ in steps of $3 \mathrm{~dB}$. The values of probability of false alarm are fixed as $0.1,0.01$, and 0.001 , respectively.

In the three cases, the probability of detection $\left(P_{D}\right)$ is close to 1 at an SNR greater than $0 \mathrm{~dB}$. The detection probability is increased by a higher value of probability of false alarm. With $\alpha=0.1$, the probability of detection is close to 1 for an SNR of $-6 \mathrm{~dB}$. Moreover, the derived theoretical $P_{D}$ is close to the simulated value.

6.2. Influence of the Frequency Estimation Error. Figure 4 shows the probability of detection with respect to the carrier frequency estimation error and the comparisons with the theoretically calculated values. The carrier frequency estimation error is, respectively, equal to $0.3,0.4$, and 0.5 for a sinusoid with 1024 samples and the probability of false alarm equals 0.01 . The rest of the parameter settings of the signal in the simulation remain the same as that stated in Section 6.1. The detection probability is increased by increasing the carrier frequency estimation error factor. For $\delta=0.5$ and an SNR close to $-9 \mathrm{~dB}$, the probability of detection is approximately equal to 1 .

In fact, a larger frequency estimation error factor improves the mean of the proposed statistic $U$, and the discrimination between nonnull and null is easier. In addition, it can be observed from Figure 3 that the derived theoretical $P_{D}$ is very close to the simulated values in the three cases.

6.3. Influence of the Number of Received Samples N. Figure 5 shows the detection performance of the credibility test versus the received samples of the signal and the comparisons with the theoretical calculated values. The number of samples is equal to 512, 1024, and 2048, respectively. The carrier frequency estimation error is set to 0.4 . The rest of the parameter

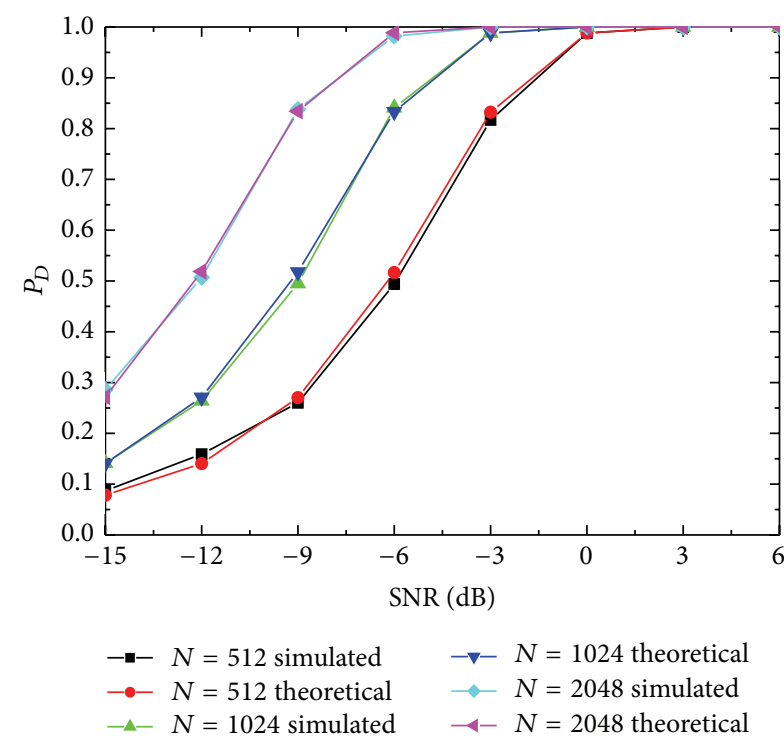

FIGURE 5: Effect of the number of received samples on the detection probability obtained by the credibility test of a frequency estimate.

settings are the same as that stated in Section 6.2. In the three cases with different sample numbers, the detection probability is close to 1 for an SNR of $0 \mathrm{~dB}$. The credibility test performance is enhanced by the greater number of received samples at the same SNR. When the number of samples is 2048, the detection probability is close to 1 for an SNR of $-6 \mathrm{~dB}$. We can observe from Figure 5 that the derived theoretical $P_{D}$ is very close to the simulated values in the three cases.

6.4. Influence of Frequency Discretization. We evaluated the performance of our method in the case of different carrier frequencies. Figure 6 shows the performance of detection versus the carrier frequency selected in the simulations. We defined the discretization factor as $d=\left(f_{0}-k_{0} \Delta F\right)$ where $k_{0}=199$ and five frequencies were generated by setting $d=0,0.1,0.3,0.5,0.7$. Clearly, if $d=0$, the frequency equals $19.53125 \mathrm{MHz}$, which is just located on the discrete frequency bin. When $d \neq 0$, it indicates that the selected frequencies are not located on the discrete frequency bin. The parameters are $N=1024, \delta=0.4, \theta=\pi / 6$, and $\alpha=0.01$. Figure 6 shows that the detection probability does not depend on the frequencies we selected by using different discretization factors, and the same levels of performance are achieved in the five cases and are approximately identical to the theoretically calculated performance in all the cases.

6.5. Influence of the Initial Phase. We investigated the impact of the initial phase on the performance of our method. Figure 7 shows the performance of detection versus the initial frequency used in the simulations. We set three initial phases as $\pi / 12, \pi / 6$, and $\pi / 3$ and the sample size as 1024 . The rest of the parameters set in each simulation are the same as that mentioned in Section 6.3. Figure 7 shows that the initial phase of the sinusoid does not affect the detection probability 


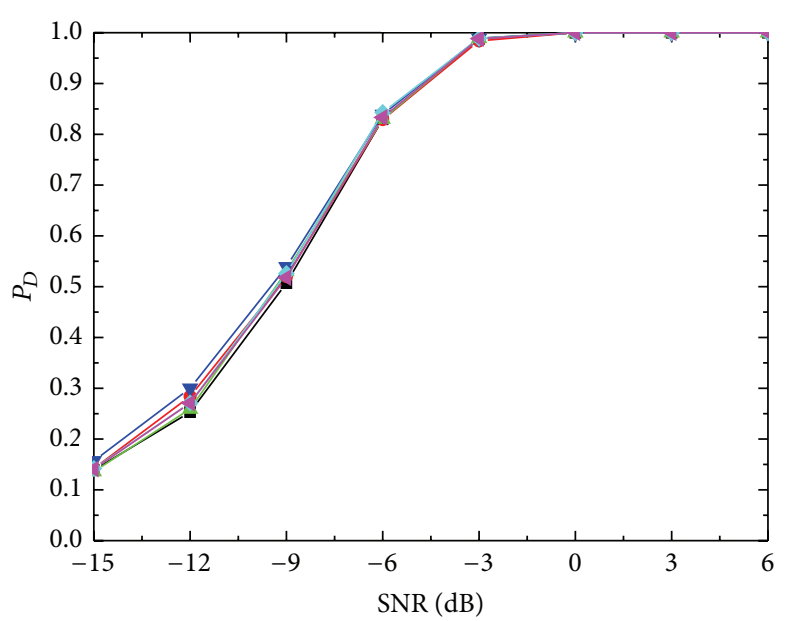

- - $f_{0}=19.599609 \mathrm{MHz}(d=0.7) \quad-\nabla-f_{0}=19.541015 \mathrm{MHz}(d=0.1)$

$\longrightarrow f_{0}=19.580078 \mathrm{MHz}(d=0.5) \multimap f_{0}=19.53125 \mathrm{MHz}(d=0)$

$-\triangle-f_{0}=19.560546 \mathrm{MHz}(d=0.3) \multimap-$ Theoretical

FIGURE 6: Effect of the frequency discretization of the signal on the detection probability obtained by the credibility test of a frequency estimate.

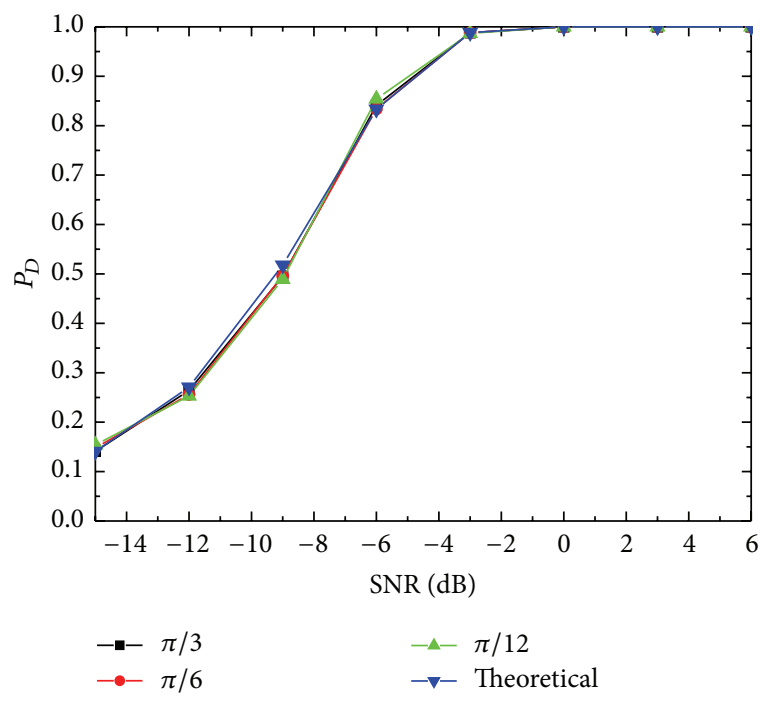

FIGURE 7: Effect of the initial phase of the signal on the detection probability obtained by the credibility test of a frequency estimate.

and the same levels of performance are achieved in the two cases and are identical to the theoretical performances as the statistic defined in (13) is not relevant to the phase because of the use of the modulus operation.

6.6. Influence of the Sampling Frequency. We now clarify the impact of the sampling frequency on the performance of the proposed method. Figure 8 shows the performance of detection versus the sampling frequency selected in the simulations. The other parameters are $f_{0}=19.081 \mathrm{MHz}$, $N=1024, \delta=0.4, \theta=\pi / 6$, and $\alpha=0.01$. From the figure, we observe that the performance of detection is not affected

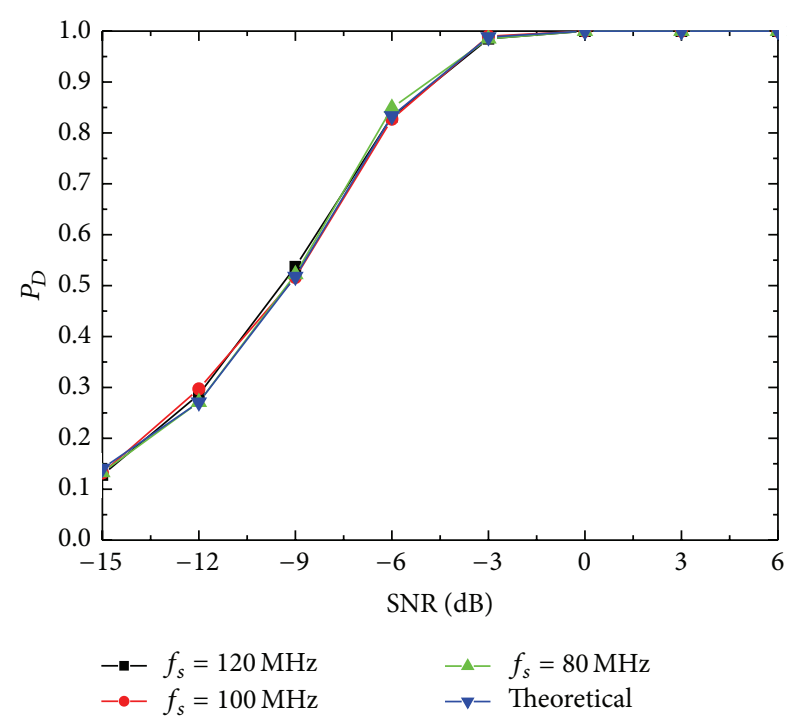

FIGURE 8: Effect of the sampling frequency of the signal on the detection probability obtained by the credibility test of a frequency estimate.

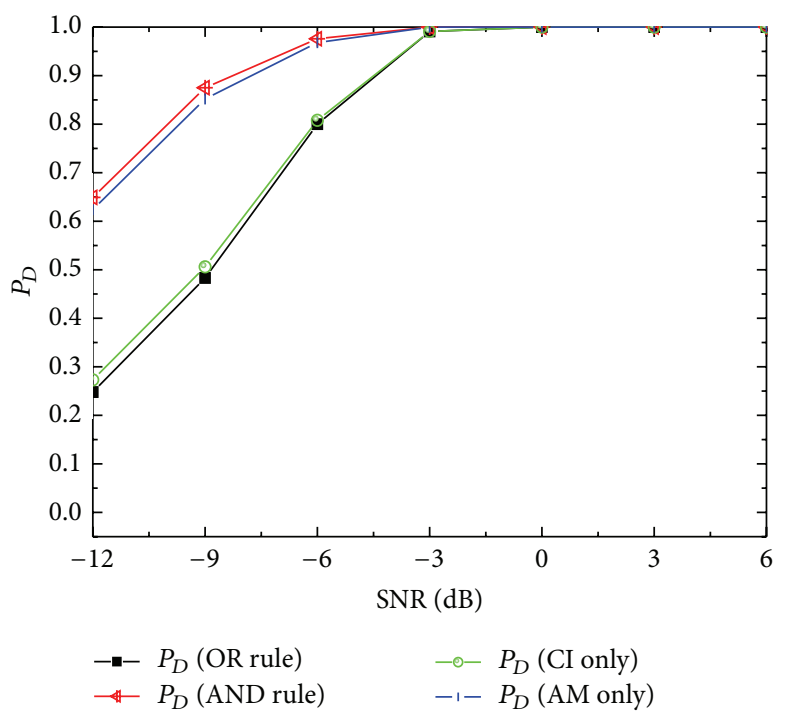

FIGURE 9: Probability of detection and probability of false alarm comparison between the proposed scheme and others method.

by the sampling frequency and is identical to the theoretical performance.

6.7. Comparison to Other Methods. Comparative experiments were also carried out for the proposed statistic, Aboutanios-Mulgrew (AM) method-based [21] detector, and a combination of them by OR and AND rules, respectively. We have described the AM-based detector that can also be used to test the credibility of the frequency estimate, in the appendix. The parameters are $f_{0}=19.081 \mathrm{MHz}$, $N=1024, \delta=0.4, \theta=\pi / 6$, and $\alpha=0.01$. The curves of probabilities of detection for different SNRs are shown in Figure 9. We can observe that, under an SNR 
less than $-3 \mathrm{~dB}$, the probabilities of detection of the AMbased method are greater than those of the CI-based method. For the fusion method, the probabilities of detection using the "AND" rule are slightly greater than those of the AMbased method, and vice versa for the "OR" rule; that is, the probability of detection is the smallest when the SNR is less than $-3 \mathrm{~dB}$. When the SNRs are greater than $-3 \mathrm{~dB}$, the probabilities of detection of the four detectors all approach 1. Therefore, under lower SNRs, the more efficient fusion methods combining the CI and AM methods need to be further developed.

It should be noted that calculating the threshold is difficult because the exact probability distribution function of the AM-based statistic $T_{\mathrm{AM}}$, defined in the appendix, is hard to be expressed analytically. Therefore, here we introduce the bootstrap-based method $[22,23]$ to obtain the threshold without depending on the analytical probability of the statistic based on AM, but the computational load increases many times because of repeated resampling operations in the bootstrap-based hypothesis test procedures.

6.8. Case Study. In this section, we use the CI-based credibility test statistic to evaluate three commonly used frequency estimators: the Rife algorithm [4], the maximum likelihood estimator [6] by Newton iteration, and the AboutaniousMulgrew estimator [21]. The carrier frequency of the sinusoid is $19.081 \mathrm{MHz}$, the initial phase is $\pi / 6$, and the sample size is 1024. The range of SNR is from $-18 \mathrm{~dB}$ to $-6 \mathrm{~dB}$. The value of probability of false alarm is set to 0.05 . If we let $D_{i}(i=$ $0,1)$ be the event associated with the decision of choosing $H_{i}(i=0,1), n_{i j}$ stands for the number of times that deciding $D_{j}$ when hypothesis $H_{i}$ is correct. Therefore, Type I error probability and Type II error probability can be calculated using $n_{01} /\left(n_{00}+n_{01}\right)$ and $n_{10} /\left(n_{10}+n_{11}\right)$, respectively. The error probability of the test can be expressed by $P_{E}=\left(n_{01}+\right.$ $\left.n_{10}\right) / 10000$, while $P_{D}=n_{11} /\left(n_{11}+n_{10}\right)$ indicates the ability of the proposed method to detect the unreliable frequency estimate, which means that the maximum absolute bias of a certain frequency estimate $(|\Delta f|)$ is less than a quarter of the discrete sampling frequency interval $(\Delta F)$.

Table 1 illustrates the performance of the credibility test for the ML frequency estimator of the sinusoid by using the proposed CI-based statistic. It should be remarked that the credibility test performance is enhanced by increasing the SNR. If the SNR is $-6 \mathrm{~dB}$, all the 10000 frequency estimates based on ML are reliable, and the error probability of the proposed test approximates to 0 . When the SNR decreases to $-12 \mathrm{~dB}, 61$ estimates are unreliable among 10000 simulations. By using the proposed statistic, all the 61 unreliable estimates can be detected, indicating a detection rate of about $100 \%$. Among the 9939 reliable estimates, there are 21 times when estimates are mistakenly decided as unreliable; in other words, the error probability is about $0.21 \%$. When the SNR is decreased to $-15 \mathrm{~dB}$, the error probability is about $0.81 \%$ and the detection rate is greater than $95 \%$.

Table 2 depicts the performance of the credibility test for the Rife-based sinusoid frequency estimator. The credibility test performance is enhanced by a larger SNR. For an SNR of $-6 \mathrm{~dB}$, all the 10000 times, the frequency estimates obtained
TABLE 1: Credibility test performance of ML frequency estimator.

\begin{tabular}{ccccccc}
\hline $\begin{array}{l}\text { SNR } \\
(\mathrm{dB})\end{array}$ & $n_{00}$ & $n_{01}$ & $n_{11}$ & $n_{10}$ & $P_{E}$ & $P_{D}$ \\
\hline-18 & 6962 & 0 & 919 & 2119 & 0.2119 & 0.302502 \\
-17 & 8304 & 12 & 1233 & 451 & 0.0463 & 0.732185 \\
-16 & 9141 & 23 & 779 & 57 & 0.008 & 0.931818 \\
-15 & 9521 & 61 & 398 & 20 & 0.0081 & 0.952153 \\
-12 & 9918 & 21 & 61 & 0 & 0.0021 & 1.000000 \\
-9 & 9988 & 5 & 7 & 0 & 0.0005 & 1.000000 \\
-6 & 10000 & 0 & 0 & 0 & 0 & $/$ \\
\hline
\end{tabular}

TABLE 2: Credibility test performance of Rife frequency estimator.

\begin{tabular}{ccccccc}
\hline $\begin{array}{l}\text { SNR } \\
(\mathrm{dB})\end{array}$ & $n_{00}$ & $n_{01}$ & $n_{11}$ & $n_{10}$ & $P_{E}$ & $P_{D}$ \\
\hline-18 & 6834 & 1 & 1195 & 1970 & 0.1971 & 0.377567 \\
-17 & 8212 & 9 & 1349 & 430 & 0.0439 & 0.758291 \\
-16 & 9041 & 33 & 831 & 95 & 0.0128 & 0.897408 \\
-15 & 9489 & 40 & 426 & 45 & 0.0085 & 0.904459 \\
-12 & 9904 & 25 & 70 & 1 & 0.0026 & 0.985915 \\
-9 & 9987 & 8 & 5 & 0 & 0.0008 & 1.000000 \\
-6 & 10000 & 0 & 0 & 0 & 0 & $/$ \\
\hline
\end{tabular}

TABLE 3: Credibility test performance of Aboutanious-Mulgrew estimator.

\begin{tabular}{ccccccc}
\hline $\begin{array}{l}\text { SNR } \\
(\mathrm{dB})\end{array}$ & $n_{00}$ & $n_{01}$ & $n_{11}$ & $n_{10}$ & $P_{E}$ & $P_{D}$ \\
\hline-18 & 7386 & 0 & 224 & 2390 & 0.239 & 0.085692 \\
-17 & 8803 & 11 & 650 & 536 & 0.0547 & 0.548061 \\
-16 & 9515 & 38 & 358 & 89 & 0.0127 & 0.800895 \\
-15 & 9850 & 50 & 81 & 19 & 0.0069 & 0.81 \\
-12 & 9966 & 34 & 0 & 0 & 0 & $/$ \\
-9 & 9994 & 6 & 0 & 0 & 0 & $/$ \\
-6 & 10000 & 0 & 0 & 0 & 0 & $/$ \\
\hline
\end{tabular}

by using the Rife algorithm are reliable $\left(H_{0}\right)$ and the error probability is approximately equal to 0 . When the SNR declines to $-12 \mathrm{~dB}$, there are 71 unreliable estimates among 10000 simulations. By using the CI-based credibility test statistic, these 70 unreliable estimates can be detected and the detection rate is about $98.6 \%$. On the other hand, among the 9929 reliable estimates, there are 25 times when the estimates are mistakenly decided as unreliable; that is, the error probability is about $0.26 \%$. With the SNR decreased to $-15 \mathrm{~dB}$, the error probability is about $0.85 \%$ and the detection rate is greater than $90 \%$.

Table 3 provides the performance of the credibility test for the AM-based sinusoid frequency estimator. The credibility test performance is enhanced by a larger SNR. Similar to that data in Tables 1 and 2, the test performance is improved by a larger SNR. With the SNR decreased to $-15 \mathrm{~dB}$, the error probability is about $0.69 \%$ and the detection rate is greater than $80 \%$. 
Overall, from Tables 2 and 3, we can observe that the proposed credibility test statistic can be used for effectively identifying unreliable frequency estimates by using ML, AM, and Rife algorithms with a high detection probability and a low error probability when the SNR is greater than $-15 \mathrm{~dB}$. Compared to the estimator performances summarized in Tables 1 and 2, the estimator performance summarized in Table 3 is the lowest because AM-based estimation has a better performance than that of both ML and Rife algorithms and the frequency estimation factors are small.

In practice, for the detected unreliable estimate, we can abandon the results or estimate the frequency of the signal again, thereby enhancing the reliability of the entire signal processing unit. For example, if the FFT-based coarse frequency estimator is used in practice and we set the parameters as $f_{s}=100 \mathrm{MHz}, f_{0}=19.55 \mathrm{MHz}, N=1000$, and the number of DFT $M=1000$, there are two cases.

(1) Case 1. When the SNRs (e.g., SNR $=-23 \mathrm{~dB}$ ) are below the SNR threshold, the maximum spectrum line cannot be detected correctly. In this case, the estimate error is larger than $0.25 \Delta F$ and the estimate will be judged as incredible by the proposed CI-based statistic. If we perform resampling over the frequency grid by setting $M=2000$, the maximum spectrum line still cannot be detected. As the absolute estimation errors are the same under the two scenarios, the resampling action cannot enhance the credibility of the estimate and this kind of incredible estimate should be abandoned.

(2) Case 2. When an SNR is greater than the SNR threshold, an incredible frequency estimate is detected because of the displacement of the maximum spectrum line by the discretization. In this case, if we enlarge $M$ to 2000 by resampling in the frequency domain, $f_{0}$ can be just moved to the discrete frequency bin. Hence, the estimation error can be decreased and the credibility can be enhanced. However, in practice, the true frequency cannot be known by the user; hence it is difficult to adjust the resampling parameter to match the estimation exactly. In this case, we need to select the more accurate estimating method or abandon the result obtained by original estimation.

\section{Conclusion}

The paper presented a CI-based credibility test algorithm for frequency estimation of a sinusoid. A credibility assessment testing model is defined to analyze the mathematical characteristics of the correlations between the observed signal and the reference signal under different hypotheses. The test is performed with the proposed threshold that is based on CI. Experimental results revealed that a good performance was achieved even at low SNRs. Credibility tests were performed with the ML frequency estimator using the proposed threshold based on CI as well with the Rife frequency estimator and the Aboutanious-Mulgrew estimator. Experimental results revealed that a good performance was achieved by the proposed method even at low SNRs.

\section{Appendix}

The frequency estimation credibility test method based on the AM estimator includes

(1) estimation of the frequency of the signal by using a certain estimation method,

(2) construction of the reference signal $y(n)$ with (3) by using the estimated frequency $\widehat{f}_{0}$,

(3) computation of the correlation between the received signal and the reference signal and estimation of the frequency displacement $\Delta \widehat{f}$ by the AboutaniosMulgrew frequency estimator [21],

(4) evaluation of the credibility of frequency estimation by comparing the statistic $T_{\mathrm{AM}}=|| \Delta \widehat{f}|-0.25 \Delta F|$ to the threshold th that can be calculated by a given false alarm $\alpha$. The hypothesis $H_{0}$ is chosen if $T_{\mathrm{AM}}<$ th. Otherwise $H_{1}$ is accepted.

\section{Conflict of Interests}

The authors declare that there is no conflict of interests regarding the publication of this paper.

\section{Acknowledgments}

This study is financially supported by the Natural Science Foundation of Jiangsu Province (Project no. BK2011837), the National Natural Science Foundation of China, 2012 (Project no. 61201208), and the 333 Research Project of Jiangsu Province (Project no. BRA2013171).

\section{References}

[1] D. C. Rife and R. R. Boorstyn, "Single-tone parameter estimation from discrete-time observation," IEEE Transactions on Information Theory, vol. 20, no. 5, pp. 591-598, 1974.

[2] S. Peleg and B. Porat, "Linear FM signal parameter estimation from discrete-time observations," IEEE Transactions on Aerospace and Electronic Systems, vol. 27, no. 4, pp. 607-616, 1991.

[3] M. Ghogho, A. Swami, and T. Durrani, "Blind estimation of frequency offset in the presence of unknown multipath," in Proceedings of the IEEE International Conference on Personal Wireless Communications, pp. 104-108, December 2000.

[4] D. C. Rife and G. A. Vincent, "Use of the discrete Fourier transform in the measurement of frequencies and levels of tones," The Bell System Technical Journal, vol. 49, no. 2, pp. 197228, 1970.

[5] J.-R. Liao and C.-M. Chen, "Phase correction of discrete Fourier transform coefficients to reduce frequency estimation bias of single tone complex sinusoid," Signal Processing, vol. 94, no. 1, pp. 108-117, 2014.

[6] T. Abatzoglou, "A fast maximum likelihood algorithm for frequency estimation of a sinusoid based on Newton's method," 
IEEE Transactions on Acoustics, Speech and Signal Processing, vol. 33, no. 1, pp. 77-89, 1985.

[7] Y. Liu, Z. Nie, Z. Zhao, and Q. H. Liu, "Generalization of iterative Fourier interpolation algorithms for single frequency estimation," Digital Signal Processing, vol. 21, no. 1, pp. 141-149, 2011.

[8] G. Wei, C. Yang, and F.-J. Chen, "Closed-form frequency estimator based on narrow-band approximation under noisy environment," Signal Processing, vol. 91, no. 4, pp. 841-851, 2011.

[9] C. Yang and G. Wei, "A noniterative frequency estimator with rational combination of three spectrum lines," IEEE Transactions on Signal Processing, vol. 59, no. 10, pp. 5065-5070, 2011.

[10] Ç. Candan and S. Koç, "Beamspace approach for detection of the number of coherent sources," in Proceedings of the IEEE Radar Conference: Ubiquitous Radar (RADAR '12), pp. 09130918, May 2012.

[11] Y. Cao, G. Wei, and F.-J. Chen, "A closed-form expanded autocorrelation method for frequency estimation of a sinusoid," Signal Processing, vol. 92, no. 4, pp. 885-892, 2012.

[12] F. Qian, S. Leung, Y. Zhu, W. Wong, D. Pao, and W. Lau, "Damped sinusoidal signals parameter estimation in frequency domain," Signal Processing, vol. 92, no. 2, pp. 381-391, 2012.

[13] G. Richard, ELINT: The Interception and Analysis of Radar Signals, John Wiley \& Sons, Artech House, Dedham, Mass, USA, 2nd edition, 2006.

[14] W. Su, J. A. Kosinski, and M. Yu, "Dual-use of modulation recognition techniques for digital communication signals," in Proceedings of the IEEE Long Island Systems, Applications and Technology Conference (LISAT '06), pp. 1-6, Long Island, NY, USA, May 2006.

[15] L. Pucker, "Review of contemporaryspectrum sensing technologies," IEEE-SA P1900.6 Standards Group, 2009, http:// grouper.ieee.org/groups/dyspan/6/documents/white_papers/ P1900.6_Sensor_Survey.pdf.

[16] A. Fehske, J. Gaeddert, and J. H. Reed, "A new approach to signal classification using spectral correlation and neural networks," in Proceedings of the 1st IEEE International Symposium on New Frontiers in Dynamic Spectrum Access Networks (DySPAN '05), pp. 144-150, Baltimore, Md, USA, November 2005.

[17] W. S. Lin and K. J. R. Liu, "Modulation forensics for wireless digital communications," in Proceedings of the IEEE International Conference on Acoustics, Speech and Signal Processing (ICASSP '08), pp. 1789-1792, April 2008.

[18] G.-B. Hu, L.-Z. Xu, and M. Jin, "Reliability testing for blind processing results of LFM signals based on NP criterion," Acta Electronica Sinica, vol. 41, no. 4, pp. 739-743, 2013.

[19] A. D. Whalen, Detection of Signals in Noise, Academic Press, New York, NY, USA, 2nd edition, 1995.

[20] M. Sankaran, "On the non-central chi-square distribution," Biometrika, vol. 46, no. 1-2, pp. 235-237, 1959.

[21] E. Aboutanios and B. Mulgrew, "Iterative frequency estimation by interpolation on Fourier coefficients," IEEE Transactions on Signal Processing, vol. 53, no. 4, pp. 1237-1242, 2005.

[22] A. M. Zoubir and D. R. Iskander, Bootstrap Techniques for Signal Processing, Cambridge University Press, Cambridge, UK, 2004.

[23] A. M. Zoubir and D. R. Iskandler, "Bootstrap methods and applications," IEEE Signal Processing Magazine, vol. 24, no. 4, pp. 10-19, 2007. 


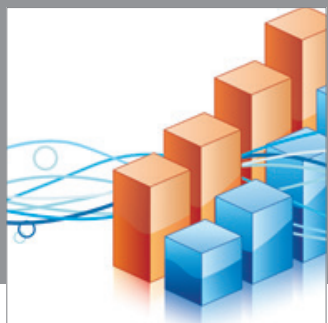

Advances in

Operations Research

mansans

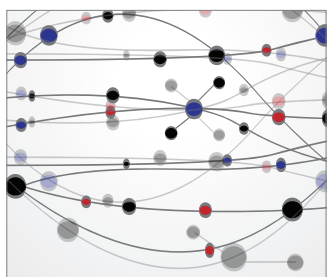

The Scientific World Journal
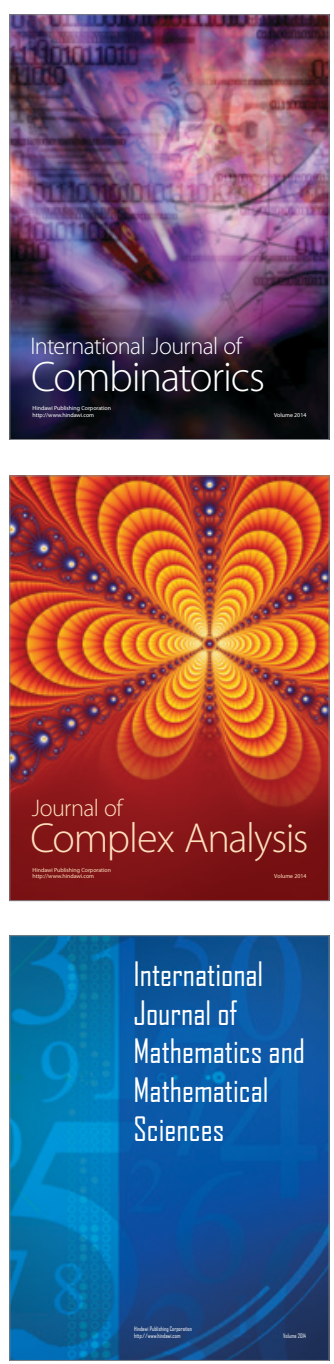
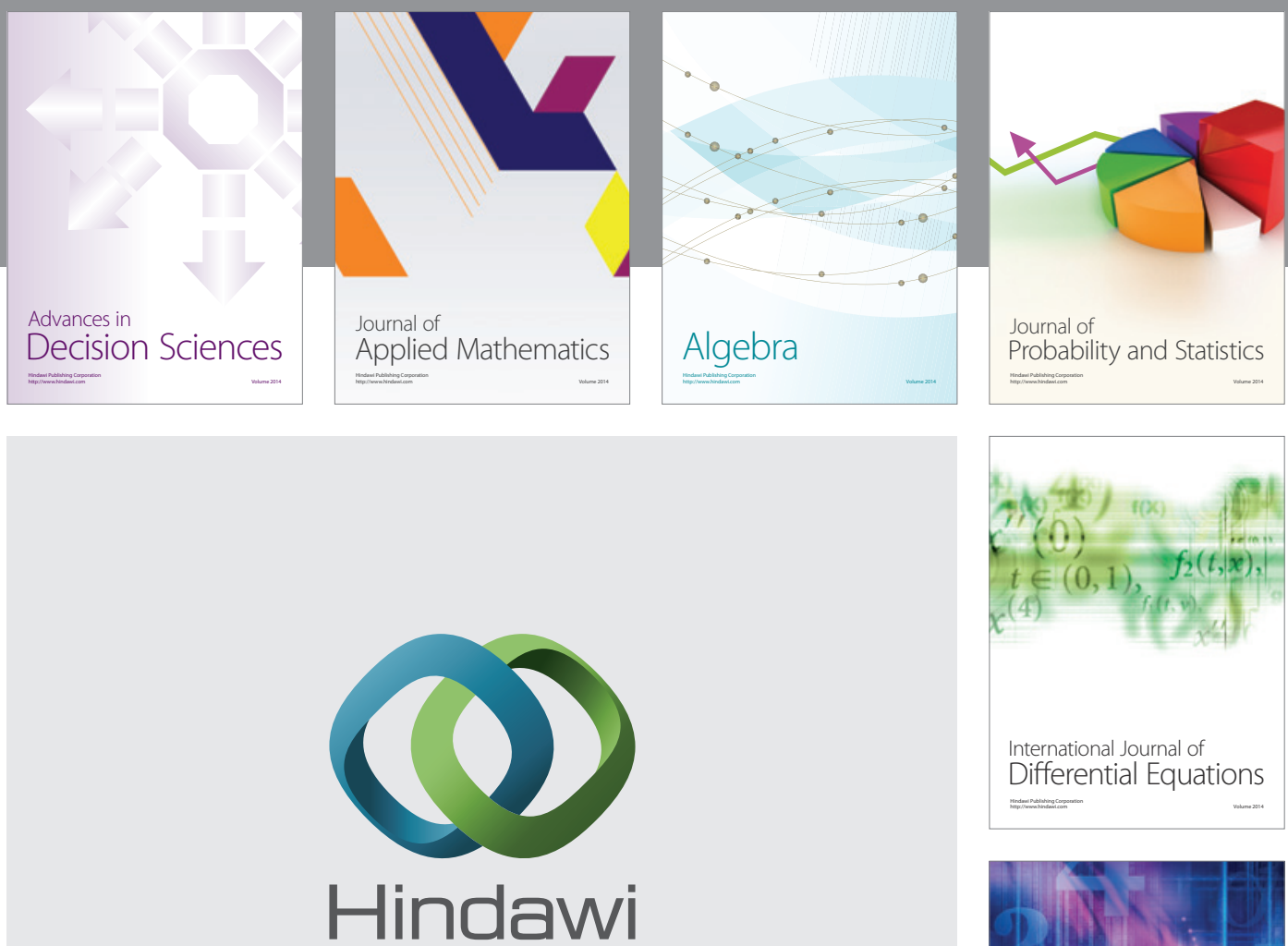

Submit your manuscripts at http://www.hindawi.com
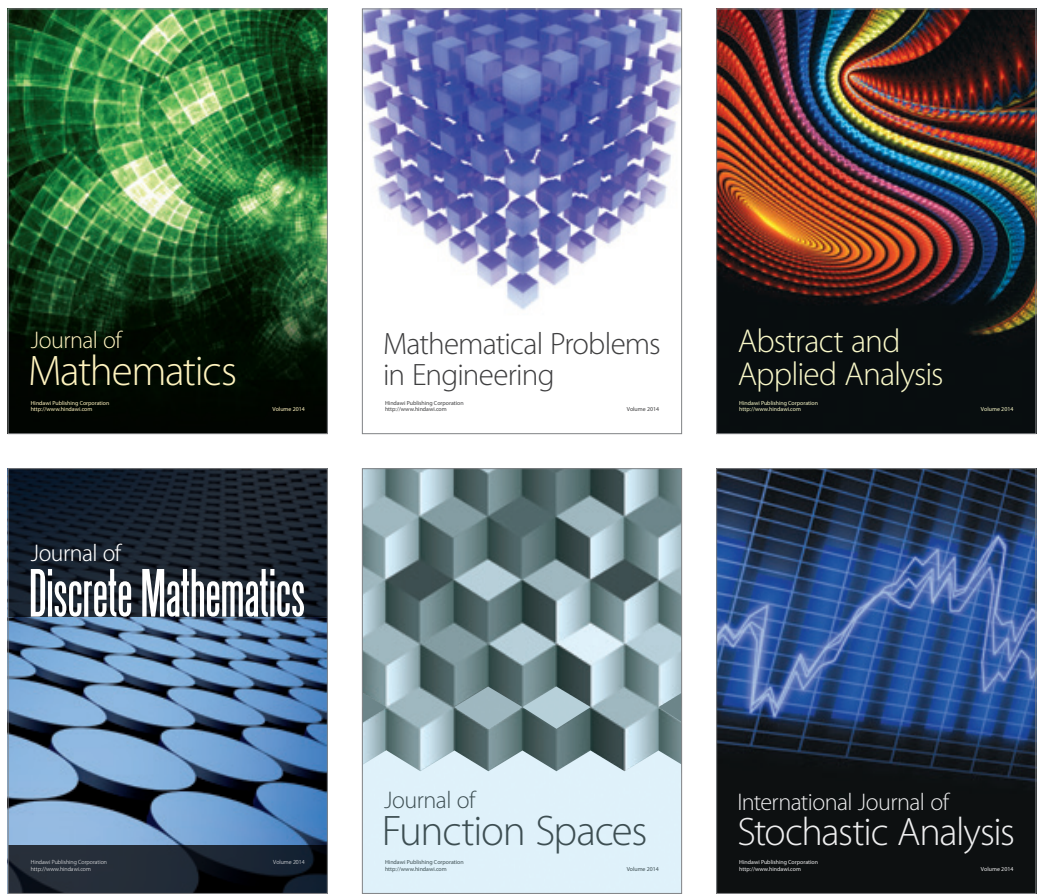

Journal of

Function Spaces

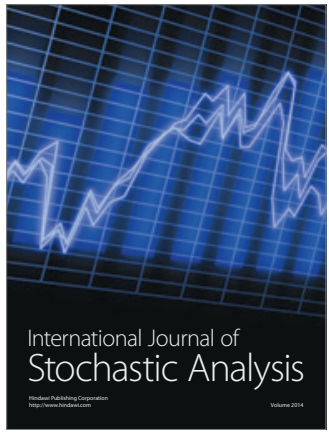

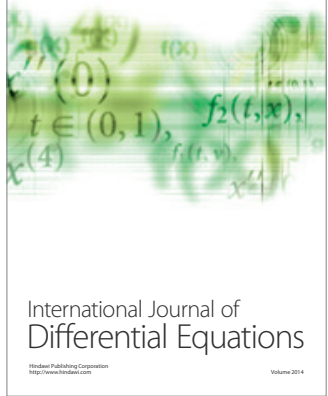
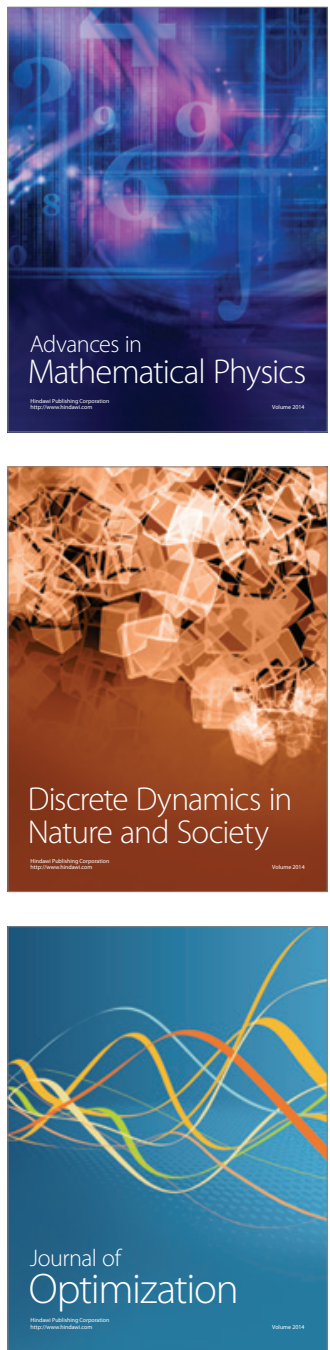